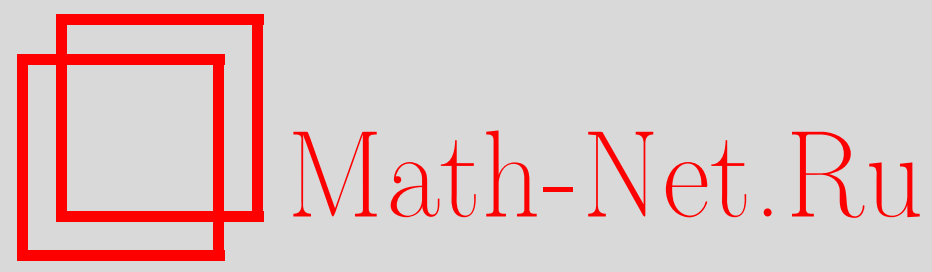

А. А. Арсеньев, Математическая модель резонансного рассеяния, Матем. заметки, 2001, том 70, выпуск 4, 491-502

DOI: https://doi.org/10.4213/mzm762

Использование Общероссийского математического портала Math-Net.Ru подразумевает, что вы прочитали и согласны с пользовательским соглашением http://www . mathnet.ru/rus/agreement

Параметры загрузки:

IP : 3.80 .253 .173

26 апреля 2023 г., 13:05:39

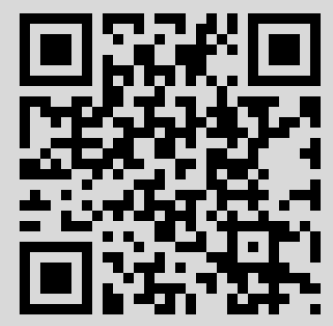




\title{
МАТЕМАТИЧЕСКАЯ МОДЕЛЬ РЕЗОНАНСНОГО РАССЕЯНИЯ
}

\author{
А. А. Арсеньев
}

\begin{abstract}
В статье предложена математическая модель возникновения скачков амплитуды рассеяния вблизи полюса матрицы рассеяния с малой мнимой частью.

Библиограффия: 7 названий.
\end{abstract}

1. Введение. В математической теории рассеяния под резонансом обычно понимают имеющий малую мнимую часть полюс функции Грина. В теоретической физике под резонансом понимают полюс аналитического продолжения матрищы рассеяния. Экспериментаторы под резонансом понимают резкое изменение измеряемых величин (сечений рассеяния) вблизи пороговых значений энергии. Хорошо известно, что связь между разньми понятиями резонанса не проста: полюс функции Грина может не быть полюсом матрицы рассеяния, полюс матрицы рассеяния может не порождать скачок амплитуды рассеяния, а скачок амплитуды рассеяния может не быть связан с полюсом матрицы рассеяния. Обсуждение этих проблем можно найти в [1]. В нашей работе в рамках теории рассеяния Бирмана-Като [2], [3] мы предлагаем математическую модель, в которой доказывается существование полюса функции Грина, доказывается, что этот полюс есть полюс матрицы рассеяния, и доказьвается, что вблизи полюса амплитуда рассеяния испытьвает скачок. В предлагаемой модели под резонансом понимается возмушенное собственное значение, которое до возмущения лежало в непрерьвном спектре. М. С. Лифшицем построена теория резонансов, понимаемых как возмущенное собственное значение из непрерьвного спектра (современное изложение теории Лифшица есть в [4]). Наш подход отличается от подхода Лифшица выбором исходных пар операторов (подробные пояснения даны в конще работы).

Наши обозначения стандартны, но, несколько отступая от традиций математической теории рассеяния, невозмущенньй оператор мы обозначаем символом $A_{0}$, а возмущенный - символом $A, A=A_{0}+V$. Это связано с тем, что для выполнения наложенных нами требований на исходные пары операторов в стандартной ситуации нужно воспользоваться принципом инвариантности и взять подходящие функции от операторов эволюции.

2. Описание модели. Для упрощения изложения мы не будем стремиться к независимости всех предположений.

Пусть $H, H_{ \pm}$- такие гильбертовы пространства, что $H_{-} \subset H \subset H_{+}, H_{-}$плотно в $H$, а $H$ плотно в $H_{+} \cdot$ Пусть $\langle\cdot, \cdot\rangle,\|\cdot\|$ - скалярное произведение и норма в $H$. Мы 
предполагаем, что скалярное произведение в гильбертовом пространстве линейно по второму аргументу. Мы предполагаем, что для любого $f \in H_{-}$линейньй функционал

$$
H \ni g \rightarrow\langle f, g\rangle
$$

может быть по непрерьвности продолжен до линейного функционала на $H_{+}$и продолжение линейной формы (1) задает общий вид линейного функционала на $H_{+}$, т.е. форма $\langle\cdot, \cdot\rangle$ приводит $H_{-}$и $H_{+}$в двойственность. Символом $A^{*}$ мы обозначим оператор, сопряженньй оператору $A$ относительно формы $\langle\cdot, \cdot\rangle$.

Пусть $\Omega$-компактное топологическоепространство, $d \omega$-вероятностная борелевская мерана $\Omega$ и $L^{2}(\Omega, d \omega)$ - гильбертово пространство интегрируемых с квадратом функций на $\Omega$. Скалярное произведение в $L^{2}(\Omega, d \omega)$ мы обозначим символом $(\cdot, \cdot)$.

Пусть $h$ - гильбертово пространство интегрируемых с квадратом функций на $(a ; b)$ со значениями в $L^{2}(\Omega, d \omega)$,

$$
\langle f, g\rangle_{h}=\int_{a}^{b}(f(\lambda), g(\lambda)) d \lambda, \quad f(\lambda), g(\lambda) \in L^{2}(\Omega, d \omega) .
$$

Пусть $A_{0}$ - ограниченньй самосопряженньй оператор в $H$, которьй унитарно эквивалентен оператору умножения на $\lambda$ в пространстве $h$, т.е. пусть существует такой оператор $U \in L(H \rightarrow h)$ (символом $L\left(B_{1} \rightarrow B_{2}\right)$ мы обозначаем банахово пространство линейных непрерывных отображений из банахова пространства $B_{1}$ в банахово пространство $\left.B_{2}\right)$, что

$$
\begin{aligned}
\langle f, g\rangle & =\langle U f, U g\rangle_{h}, \\
\left\langle f, A_{0} g\right\rangle & =\int_{a}^{b} \lambda(U(f)(\lambda), U(g)(\lambda)) d \lambda .
\end{aligned}
$$

Мы предположим, что обратный оператор $U^{-1}$ существует, ограничен и в пространстве $h$ действует как интегральньй оператор с ядром $U^{-1}(\lambda, \omega)$, причем функция

$$
(a ; b) \otimes \Omega \ni(\lambda \otimes \omega) \rightarrow U^{-1}(\lambda, \omega) \in H_{+}
$$

аналитична по $\lambda$ при фиксированном $\omega$ в не зависящей от $\omega$ комплексной окрестности некоторого интервала $\left(\lambda_{\infty}-\delta ; \lambda_{\infty}+\delta\right)$ и непрерьвна по совокупности переменньх $(\lambda \otimes \omega)$ как функция со значениями в $H_{+}$. Мы предполагаем, что для любого $\psi \in H_{-}$в не зависящей от $\psi$ комплексной окрестности интервала $\left(\lambda_{\infty}-\delta ; \lambda_{\infty}+\delta\right)$ функция

$$
\lambda \rightarrow U(\psi)(\lambda) \in C(\Omega)
$$

аналитична по $\lambda$ как функция со значениями в $C(\Omega)$.

Мы предположим, что в комплексной окрестности интервала $\left(\lambda_{\infty}-\delta ; \lambda_{\infty}+\delta\right)$ функция

$$
\lambda \rightarrow R\left(\lambda, A_{0}\right)=\left(\lambda E-A_{0}\right)^{-1} \in L(H \rightarrow H)
$$

как функция со значениями в $L\left(H_{-} \rightarrow H_{+}\right)$имеет аналитическое продолжение из верхней полуплоскости в нижнюю (это продолжение мы обозначим символом $R^{+}\left(\lambda, A_{0}\right)$ ) и из нижней полуплоскости в верхнюю (это продолжение мы обозначим символом $\left.R^{-}\left(\lambda, A_{0}\right)\right)$. 
Мы говорим, что оператор $V \in L(H \rightarrow H)$ допустим, если это самосопряженньй ядерньй в $H$ оператор, который по непрерьвности может быть продолжен на $H_{+}$, удовлетворяет условию $V H_{+} \subset H_{-}$и вполне непрерывен как оператор из $L\left(H_{+} \rightarrow H_{-}\right)$.

Пусть $V_{n}$ - последовательность допустимых операторов, которая сходится к допустимому оператору $V_{\infty}$ в ядерной топологии пространства $L(H \rightarrow H)$ и в равномерной операторной топологии пространства $L\left(H_{+} \rightarrow H_{-}\right)$.

Определим операторы $A_{n}$ формулой

$$
A_{n}=A_{0}+V_{n}, \quad n \leqslant \infty .
$$

В дальнейшем величины, относяшиеся к операторам $A_{n}, V_{n}, n \leqslant \infty$, мы будем помечать соответствуюшим индексом, который может быть опущен в тех формулах, где индекс может принимать любое достаточно большое значение, включая бесконечность.

Пусть $\psi_{\infty}$ - собственная функция оператора $A_{\infty}$ в пространстве $H$ :

$$
A_{\infty} \psi_{\infty}=\lambda_{\infty} \psi_{\infty}, \quad\left\|\psi_{\infty}\right\|=1 .
$$

Точка $\lambda_{\infty}$ в (2) - это та точка, о которой шла речь выше.

Мы предположим, что

$$
\psi_{\infty} \in H_{-} .
$$

Помимо удовлетворяюшей условию (3) функции равенству (2) могут удовлетворять и другие функции; для дальнейшего это не существенно.

Определим операторы

$$
\Gamma^{ \pm}(\lambda)=R^{ \pm}\left(\lambda, A_{0}\right) V .
$$

Из наших предположений следует, что функция $\Gamma^{ \pm}(\lambda)$ аналитична по $\lambda$ в окрестности интервала $\left(\lambda_{\infty}-\delta ; \lambda_{\infty}+\delta\right)$ как функция со значениями в пространстве $L\left(H_{+} \rightarrow H_{+}\right)$.

Из (2) и условия (3) следует, что

$$
\psi_{\infty}=\Gamma_{\infty}^{ \pm}(\lambda) \psi_{\infty}
$$

Мы предположим, что в пространстве $H_{+}$вьполнены условия

$$
\begin{aligned}
\operatorname{Ker}\left(E-\Gamma_{\infty}^{ \pm}\left(\lambda_{\infty}\right)\right) & \subset H_{-}, \\
\operatorname{Ker}\left(E-\Gamma_{\infty}^{+}\left(\lambda_{\infty}\right)\right) & =\operatorname{Ker}\left(E-\Gamma_{\infty}^{-}\left(\lambda_{\infty}\right)\right), \\
\operatorname{dim} \operatorname{Ker}\left(E-\Gamma_{\infty}^{ \pm}\left(\lambda_{\infty}\right)\right) & =1 .
\end{aligned}
$$

Далее мы предположим, что

$$
\left\langle\psi_{\infty}, V_{\infty} \psi_{\infty}\right\rangle \neq 0 .
$$

Мы будем назьвать регулярным собственным значением оператора $A$ то собственное значение, которое удовлетворяет перечисленньм вьше условиям для $\lambda_{\infty}$. Мы предположим, что на интервале $\left(\lambda_{\infty}-\delta ; \lambda_{\infty}+\delta\right)$ оператор $A_{n}$ не имеет собственных значений при $n<\infty$.

Нетрудно видеть, что сформулированные вьше условия являются переформулировками хорошо известных фактов теории рассеяния для возмушенного оператора Лапласа: свойств резольвенты оператора Лапласа, перобразования Фурье, леммы Реллиха.

Теперь мы сформулируем несколько специальных предположений,которые мы в дальнейшем будем считать выполненньми. 
ПРЕДПОЛОЖЕНИЕ S1. Существует гомоморфизм

$$
l: H_{+} \rightarrow H_{+}
$$

который удовлетворяет следующему условию: в некоторой комплексной окрестности точки $\mu=1$ и в некоторой комплексной окрестности интервала $\left(\lambda_{\infty}-\delta\right.$; $\left.\lambda_{\infty}+\delta\right)$ выполнены соотношения

$$
\begin{aligned}
l(\mu \psi) & =\bar{\mu}(\psi), \\
l\left(\operatorname{Ker}\left(\bar{\mu} E-\Gamma^{-}(\bar{\lambda})\right)\right) & \subset \operatorname{Ker}\left(\mu E-\Gamma^{+}(\lambda)\right), \\
l\left(\operatorname{Ker}\left(\mu E-\Gamma^{+}(\lambda)\right)\right) & \subset \operatorname{Ker}\left(\bar{\mu} E-\Gamma^{-}(\bar{\lambda})\right) .
\end{aligned}
$$

ПРЕДПОЛОЖЕНИЕ S2. Существует непрерывное отображсние

$$
p: \Omega \rightarrow \Omega
$$

такое, что в некоторой комплексной окрестности точки $\mu=1$ и в некоторой комплексной окрестности интервала $\left(\lambda_{\infty}-\delta ; \lambda_{\infty}+\delta\right)$ выполнено равенство

$$
\left|\left\langle V l^{-1}(\psi), U^{-1}(\lambda, p(\omega))\right\rangle\right|=|U(V \psi)(\lambda, \omega)| \quad \forall \omega \in \Omega, \quad \forall \psi \subset \operatorname{Ker}\left(\mu E-\Gamma^{ \pm}(\lambda)\right) .
$$

Из (6) следует, что

$$
l\left(\psi_{\infty}\right)=\beta \psi_{\infty}, \quad \beta \neq 0
$$

В рассмотренных нами задачах потенциального рассеяния и дифракции отображение $l$ есть комбинация комплексного сопряжения и пространственной симметрии, отображение $p$ есть отображение единичной сферы в себя и $|\beta|=1$.

3. Свойства модели. Изучим свойства описанной вьше модели.

ЛЕмма 1. Если $\psi_{\infty}$ удовлетворяет условию (7), то число $\mu=1$ есть полупростое собственное значение оператора $\Gamma_{\infty}^{ \pm}\left(\lambda_{\infty}\right)$ в пространстве $H_{+}$.

ДокАЗАТЕЛЬСтво. Если $\varphi \in H_{+}$, то функция

$$
\lambda \rightarrow\left\langle\left(\lambda E-A_{0}\right) \psi_{\infty}, R\left(\lambda, A_{0}\right) V_{\infty} \varphi\right\rangle
$$

аналитична в окрестности $\lambda_{\infty}$, а так как

$$
\left\langle\left(\lambda E-A_{0}\right) \psi_{\infty}, R\left(\lambda, A_{0}\right) V_{\infty} \varphi\right\rangle=\left\langle\psi_{\infty}, V_{\infty} \varphi\right\rangle, \quad \operatorname{Im} \lambda>0
$$

то

$$
\left\langle\left(\lambda_{\infty} E-A_{0}\right) \psi_{\infty}, \Gamma^{+}\left(\lambda_{\infty}\right) \varphi\right\rangle=\left\langle\psi_{\infty}, V_{\infty} \varphi\right\rangle
$$

Если $\varphi$ есть решение уравнения

$$
\left(E-\Gamma^{+}\left(\lambda_{\infty}\right)\right) \varphi=\psi_{\infty},
$$

то

$$
\begin{aligned}
\left\langle\left(\lambda_{\infty} E-A_{0}\right) \psi_{\infty},\left(E-\Gamma^{+}\left(\lambda_{\infty}\right)\right) \varphi\right\rangle & =\left\langle V_{\infty} \psi_{\infty}, \psi_{\infty}\right\rangle \\
& =\left\langle V_{\infty} \psi_{\infty}, \varphi\right\rangle-\left\langle\psi_{\infty}, V_{\infty} \varphi\right\rangle=0
\end{aligned}
$$


что противоречит (7). Следовательно, уравнение (13) решений не имеет, и все нильпотенты оператора $\Gamma_{\infty}^{+}\left(\lambda_{\infty}\right)$ равны нулю.

Аналогичное лемме 1 утверждение доказано в [5].

Из леммы 1 следует, что существуют числа $\varepsilon>0, \delta>0$ такие, что при $n=\infty$ проектор

$$
P_{n}^{+}(\lambda)=\frac{1}{2 \pi i} \oint_{|1-\mu|=\varepsilon} R\left(\mu, \Gamma_{n}^{+}(\lambda)\right) d \mu, \quad\left|\lambda-\lambda_{\infty}\right|<\delta,
$$

одномерен, аналитически зависит от $\lambda$ и удовлетворяет уравнению

$$
\Gamma_{n}^{+}(\lambda) P_{n}^{+}(\lambda)=P_{n}^{+}(\lambda) \Gamma_{n}^{+}(\lambda)=\mu_{n}^{+}(\lambda) P_{n}^{+}(\lambda) .
$$

Действительно, так как при $n \rightarrow \infty$ равномерно по $\lambda$

$$
\Gamma_{n}^{+}(\lambda) \rightarrow \Gamma_{\infty}^{+}(\lambda)
$$

то при $n \rightarrow \infty$

$$
P_{n}^{+}(\lambda) \rightarrow P_{\infty}^{+}(\lambda)
$$

и поэтому при $n \geqslant n_{0}$ проектор $P_{n}^{+}(\lambda)$ одномерен и удовлетворяет уравнению $(15)$, где функция $\lambda \rightarrow \mu_{n}^{+}(\lambda)$ аналитична по $\lambda$ в окрестности точки $\lambda_{\infty}$.

В дальнейшем число $n$ предполагается достаточно большим, а число $\delta$ достаточно малым.

Лемма 2. Определенный формулой (14) проектор $P_{n}^{+}(\lambda)$ можсет быть вычислен по формуле

$$
P^{+}(\lambda) f=c(\lambda)\left\langle V l^{-1}(\psi(\lambda)), f\right\rangle
$$

$2 \partial e$

$$
\begin{aligned}
\mu^{+}(\lambda) \psi(\lambda) & =\Gamma^{+}(\lambda) \psi(\lambda), \quad \psi \in H_{+} \\
c(\lambda) & =\left\langle V l^{-1}(\psi(\lambda)), \psi(\lambda)\right\rangle^{-1} \\
\left|1-\mu^{+}(\lambda)\right| & <\varepsilon, \quad\left|\lambda-\lambda_{\infty}\right|<\delta .
\end{aligned}
$$

Функиии $c(\lambda), \mu^{+}(\lambda)$ аналитичны по $\lambda$ в окрестности точки $\lambda_{\infty}$ и равномерно по $\lambda$ npu $n \rightarrow \infty$

$$
c_{n}(\lambda) \rightarrow c_{\infty}(\lambda), \quad \mu_{n}^{+}(\lambda) \rightarrow \mu_{\infty}^{+}(\lambda), \quad\left\|\psi_{n}(\lambda)-\psi_{\infty}(\lambda)\right\|_{+} \rightarrow 0
$$

ДокАЗАтЕльство. Область значений проектора $P_{\infty}^{+}\left(\lambda_{\infty}\right)$ есть линейное пространство, натянутое на $\psi_{\infty}$, поэтому существует такой элемент $f_{0} \in H_{+}$, что $P_{\infty}^{+}\left(\lambda_{\infty}\right) f_{0}=$ $\psi_{\infty}$. Положим

$$
\psi_{n}(\lambda)=P_{n}^{+}(\lambda) f_{0}
$$

$\Phi$ ункция $\psi_{n}(\lambda)$ аналитична по $\lambda$ в окрестности точки $\lambda_{\infty}$ и удовлетворяет уравнению (17). Так как проектор $P_{n}^{+}(\lambda)$ одномерен, то он может быть записан в виде

$$
P_{n}^{+}(\lambda) f=\langle\varphi(\lambda), f\rangle \psi_{n}(\lambda), \quad \varphi(\lambda) \in H_{-},
$$


причем функция $\varphi(\lambda)$ аналитична по $\lambda$. Из (15) следует, что

$$
V R^{-}\left(\bar{\lambda}, A_{0}\right) \varphi(\lambda)=\bar{\mu}^{+}(\lambda) \varphi(\lambda)
$$

Определим функцию

$$
\eta(\lambda)=R^{-}\left(\bar{\lambda}, A_{0}\right) \varphi(\lambda)
$$

Из (21) следует, что

$$
\bar{\mu}^{+}(\lambda) \eta(\lambda)=\Gamma^{-}(\bar{\lambda}) \eta(\lambda)
$$

из (9) следует, что

$$
\mu^{+}(\lambda) l(\eta(\lambda))=\Gamma^{+}(\lambda) l(\eta(\lambda))
$$

Из (6) вытекают равенства

$$
l(\eta(\lambda))=\alpha \psi(\lambda), \quad \eta(\lambda)=\bar{\alpha} l^{-1}(\psi(\lambda))
$$

поэтому в (22)

$$
\varphi(\lambda)=\left(\bar{\lambda} E-A_{0}\right) \eta(\lambda)
$$

Из (23) следует, что

$$
\bar{\mu}^{+}(\lambda)\left(\bar{\lambda} E-A_{0}\right) \eta(\lambda)=V \eta(\lambda)=\bar{\alpha} V l^{-1}(\psi(\lambda))=\bar{\mu}^{+}(\lambda) \varphi(\lambda) .
$$

Следовательно,

$$
\varphi(\lambda)=\bar{c}(\lambda) V l^{-1}(\psi(\lambda)) .
$$

Коэффициент $c(\lambda)$ определяется из условия нормировки.

$\Phi$ ункцию $\lambda \rightarrow \mu_{n}^{+}(\lambda)$ в окрестности точки $\lambda_{\infty}$ мы определяем равенством (15).

Лемма 3. Справедлива формула

$$
\left.\frac{d \mu_{\infty}^{+}(\lambda)}{d \lambda}\right|_{\lambda=\lambda_{\infty}}=-\left\langle\psi_{\infty}, V_{\infty} \psi_{\infty}\right\rangle^{-1}
$$

ДокАЗАТЕЛЬСТво. Умножая уравнение $(17)$ на $\left(\lambda E-A_{0}\right)$, мы получаем

$$
\mu_{\infty}^{+}(\lambda)\left(\lambda E-A_{0}\right) \psi_{\infty}(\lambda)=V_{\infty} \psi_{\infty}(\lambda), \quad \operatorname{Im} \lambda>0
$$

Скалярно умножая это уравнение на $\psi_{\infty}\left(\lambda_{\infty}\right)$ и дифференцируя по $\lambda$, имеем

$$
\begin{aligned}
\left\langle\psi_{\infty}\left(\lambda_{\infty}\right),\left(\lambda E-A_{0}\right) \psi_{\infty}(\lambda)\right\rangle \frac{d \mu_{\infty}^{+}(\lambda)}{d \lambda}+\mu_{\infty}^{+}(\lambda)\left\langle\psi_{\infty}\left(\lambda_{\infty}\right), \psi_{\infty}(\lambda)\right\rangle \\
\quad+\mu_{\infty}^{+}(\lambda)\left\langle\psi_{\infty}\left(\lambda_{\infty}\right),\left(\lambda E-A_{0}\right) \frac{d \psi_{\infty}(\lambda)}{d \lambda}\right\rangle \\
=\left\langle\psi_{\infty}\left(\lambda_{\infty}\right), V_{\infty} \frac{d \psi_{\infty}(\lambda)}{d \lambda}\right\rangle .
\end{aligned}
$$

В этом равенстве мы положим $\lambda=\lambda_{\infty}+\varepsilon, \varepsilon \rightarrow+0$, и получим требуемую формулу. 
Из леммы 3 следует, что функция

$$
\lambda \rightarrow\left(1-\mu_{\infty}^{+}(\lambda)\right)
$$

в точке $\lambda_{\infty}$ имеет нуль первого порядка. Следовательно, при достаточно большом $n$ функция

$$
\lambda \rightarrow\left(1-\mu_{n}^{+}(\lambda)\right)
$$

в окрестности точки $\lambda_{\infty}$ имеет нуль первого порядка. Мы обозначим его через $\lambda_{n}$ :

$$
\mu_{n}^{+}\left(\lambda_{n}\right)=1
$$

Легко видеть, что число $\lambda_{n}$ при $\operatorname{Im} \lambda \geqslant 0$ есть собственное значение оператора $A_{n}$. Оператор $A_{n}$ самосопряжен и не имеет собственных значений в верхней полуплоскости. По предположению он не имеет собственных значений на интервале $\left(\lambda_{\infty}-\delta ; \lambda_{\infty}+\delta\right)$. Следовательно, $\operatorname{Im} \lambda_{n}<0, n<\infty$.

ЛЕмма 4. Если функиия $\psi(\lambda)$ удовлетворяет уравнению $(17)$, то при $\lambda \in\left(\lambda_{\infty}-\delta\right.$; $\left.\lambda_{\infty}+\delta\right)$ выполнено равенство

$$
\operatorname{Im} \mu^{+}(\lambda)\langle\psi(\lambda), V \psi(\lambda)\rangle=-\pi(U(V \psi(\lambda))(\lambda), U(V \psi(\lambda))(\lambda))
$$

ДокАЗАТЕЛЬСтво. Умножая равенство (17) в точке $\lambda+i \varepsilon$ скалярно на $V \psi(\lambda+i \varepsilon)$ и вычитая комплексно сопряженное выражение, мы получаем

$$
\begin{aligned}
& 2 i \operatorname{Im} \mu^{+}(\lambda+i \varepsilon)\langle V \psi(\lambda+i \varepsilon), \psi(\lambda+i \varepsilon)\rangle \\
& \quad=\left\langle V \psi(\lambda+i \varepsilon),\left[R\left(\lambda+i \varepsilon, A_{0}\right)-R\left(\lambda-i \varepsilon, A_{0}\right)\right] V \psi(\lambda+i \varepsilon)\right\rangle .
\end{aligned}
$$

Переходя в этом равенстве к пределу $\varepsilon \rightarrow+0$, мы получаем утверждение леммы.

Из леммы 4 вытекает, что

$$
\left\|U\left(V \psi_{\infty}\right)\left(\lambda_{\infty}\right) \mid L^{2}(\Omega, d \omega)\right\|=0
$$

В силу непрерывности по совокупности переменных функции

$$
\lambda \otimes \omega \rightarrow U\left(V \psi_{\infty}\right)(\lambda, \omega)
$$

отсюда следует, что

$$
U\left(V \psi_{\infty}\right)\left(\lambda_{\infty}, \omega\right) \equiv 0
$$

В пространстве $H_{+}$определим оператор

$$
T_{n}^{+}(\lambda)=V_{n}+V_{n} R^{+}\left(\lambda, A_{n}\right) V_{n}, \quad\left|\lambda-\lambda_{\infty}\right|<\delta, \quad \lambda \neq \lambda_{\infty} .
$$


Лемма 5. Onератор $T_{n}^{+}(\lambda)$ при достаточно больиих $n$ можсет быть записан в виде

$$
T_{n}^{+}(\lambda)=r_{n}(\lambda)+T_{n}^{+}(\lambda)_{\mathrm{reg}}
$$

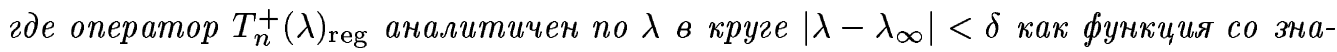
чениями в $L\left(H_{+} \rightarrow H_{+}\right)$и равномерно по $\lambda$ имеет предел при $n \rightarrow \infty$ :

$$
\lim _{n \rightarrow \infty} \sup _{\left|\lambda-\lambda_{\infty}\right|<\delta}\left\|T_{n}^{+}(\lambda)_{\mathrm{reg}}-T_{\infty}^{+}(\lambda)_{\mathrm{reg}} \mid L\left(H_{+} \rightarrow H_{+}\right)\right\|=0
$$

Oператор $r_{n}(\lambda)$ задается формулой

$$
r_{n}(\lambda) f=\frac{c_{n}(\lambda) \mu_{n}^{+}(\lambda)}{1-\mu_{n}^{+}(\lambda)}\left\langle V_{n} l^{-1}\left(\psi_{n}(\lambda)\right), f\right\rangle \psi_{n}(\lambda)
$$

ДокАЗАТЕЛЬСТво. Из второго резольвентного уравнения следует, что

$$
R^{+}\left(\lambda, A_{n}\right)=\left(E-\Gamma_{n}^{+}(\lambda)\right)^{-1} R^{+}\left(\lambda, A_{0}\right)
$$

Так как точка $\mu_{n}^{+}(\lambda)$ есть полюс первого порядка для $\left(\mu E-\Gamma_{n}^{+}(\lambda)\right)^{-1}$, то

$$
\left(E-\Gamma_{n}^{+}(\lambda)\right)^{-1}=\frac{P_{n}^{+}(\lambda)}{1-\mu_{n}^{+}(\lambda)}+S_{n}(\lambda)_{\mathrm{reg}},
$$

где оператор $S_{n}(\lambda)_{\text {reg }}$ аналитичен по $\lambda$ и при $n \rightarrow \infty$ равномерно по $\lambda$ сходится к оператору $S_{\infty}(\lambda)_{\mathrm{reg}}$. Следовательно,

$$
T_{n}^{+}(\lambda)=\frac{V_{n} P_{n}^{+}(\lambda) \Gamma_{n}^{+}(\lambda)}{1-\mu_{n}^{+}(\lambda)}+T_{n}^{+}(\lambda)_{\mathrm{reg}},
$$

где

$$
T_{n}^{+}(\lambda)_{\mathrm{reg}}=V_{n}+V_{n} S_{n}(\lambda)_{\mathrm{reg}} V_{n}
$$

В силу леммы 2 и предположения S1

$$
\begin{aligned}
V_{n} P_{n}^{+}(\lambda) \Gamma_{n}^{+}(\lambda) f & =c_{n}(\lambda)\left\langle V_{n} l^{-1}\left(\psi_{n}(\lambda)\right), R^{+}\left(\lambda, A_{0}\right) V_{n} f\right\rangle V_{n} \psi_{n}(\lambda) \\
& =c_{n}(\lambda)\left\langle R^{-}\left(\bar{\lambda}, A_{0}\right) V_{n} l^{-1}\left(\psi_{n}(\lambda)\right), V_{n} f\right\rangle V_{n} \psi_{n}(\lambda) \\
& =c_{n}(\lambda)\left\langle\Gamma_{n}^{-}(\bar{\lambda}) l^{-1}\left(\psi_{n}(\lambda)\right), V_{n} f\right\rangle V_{n} \psi_{n}(\lambda) \\
& =c_{n}(\lambda) \mu_{n}^{+}(\lambda)\left\langle V_{n} l^{-1}\left(\psi_{n}(\lambda)\right), f\right\rangle V_{n} \psi_{n}(\lambda) .
\end{aligned}
$$


4. Основные результаты. Переходим к изложению основных результатов: изучению оператора рассеяния $S\left(A_{n}, A_{0}\right)$. Как известно [2], оператор рассеяния коммутирует с $A_{0}$ и в пространстве $h$ задается операторной функцией

$$
\lambda \rightarrow S_{n}(\lambda) \in L\left(L^{2}(\Omega, d \omega) \rightarrow L^{2}(\Omega, d \omega)\right)
$$

которая в рассматриваемом случае имеет вид

$$
S_{n}(\lambda)=\mathrm{id}-2 \pi i t_{n}^{+}(\lambda)
$$

где id - единичный оператор в $L^{2}(\Omega, d \omega)$, a $t_{n}^{+}(\lambda)$ - интегральный оператор в $L^{2}(\Omega, d \omega)$ с ядром

$$
t_{n}^{+}\left(\lambda, \omega_{1}, \omega_{2}\right)=U\left(T_{n}^{+}(\lambda) U^{-1}\left(\lambda, \omega_{1}\right)\right)\left(\lambda, \omega_{2}\right)
$$

Из леммы 5 следует, что правая часть (32) может быть записана в виде

$$
\begin{aligned}
t_{n}^{+}\left(\lambda, \omega_{1}, \omega_{2}\right) & =\frac{c_{n}(\lambda) \mu_{n}^{+}(\lambda)}{1-\mu_{n}^{+}(\lambda)}\left\langle V_{n} l^{-1}\left(\psi_{n}(\lambda)\right), U^{-1}\left(\lambda, \omega_{2}\right)\right\rangle U\left(V_{n} \psi_{n}(\lambda)\right)\left(\lambda, \omega_{1}\right) \\
& =t_{n}^{+}\left(\lambda, \omega_{1}, \omega_{2}\right)_{\mathrm{reg}},
\end{aligned}
$$

где

$$
t_{n}^{+}\left(\lambda, \omega_{1}, \omega_{2}\right)_{\mathrm{reg}}=U\left(T_{n}^{+}(\lambda)_{\mathrm{reg}} U^{-1}\left(\lambda, \omega_{1}\right)\right)\left(\lambda, \omega_{2}\right)
$$

и предполагается, что либо $\lambda \in\left(\lambda_{\infty}-\delta ; \lambda_{\infty}+\delta\right), n<\infty$, либо $\lambda \neq \lambda_{\infty}$.

ЛЕмма 6. 1) Для определенной формулой (34) функции справедливо следующее утверждение:

$$
t_{n}^{+}\left(\lambda, \omega_{1}, \omega_{2}\right)_{\mathrm{reg}} \rightarrow t_{\infty}^{+}\left(\lambda, \omega_{1}, \omega_{2}\right)_{\mathrm{reg}}, \quad n \rightarrow \infty
$$

равномерно по $\lambda \in\left(\lambda_{\infty}-\delta ; \lambda_{\infty}+\delta\right), \omega_{1}, \omega_{2} \in \Omega$.

2) Для определенной формулой (33) функиии справедливо следующее утверждение:

$$
t_{n}^{+}\left(\lambda, \omega_{1}, \omega_{2}\right) \rightarrow t_{\infty}^{+}\left(\lambda, \omega_{1}, \omega_{2}\right), \quad n \rightarrow \infty,
$$

при фиксированном $\lambda \neq \lambda_{\infty}$ равномерно по $\omega_{1}, \omega_{2} \in \Omega$.

ДокАЗАТЕЛЬСТво. Эти утверждения очевидно следуют из леммы 5.

ЛЕмма 7. Если отображсение р удовлетворяет условию (11), то

$$
\lim _{\left|\lambda-\lambda_{\infty}\right| \rightarrow+0} \sup _{\omega \in \Omega}\left|t_{\infty}^{+}(\lambda, \omega, p(\omega))_{\mathrm{reg}}-t_{\infty}^{+}\left(\lambda_{\infty}, \omega, p(\omega)\right)\right|=0
$$


ДоКАЗАТЕЛЬСТво. Положим

$$
W_{n}(\lambda, \omega)=U\left(V_{n} \psi_{n}(\lambda)\right)(\lambda, \omega)
$$

Из предположения S2 и леммы 5 следует, что

$$
\left|t_{\infty}^{+}(\lambda, \omega, p(\omega))_{\mathrm{reg}}-t_{\infty}^{+}\left(\lambda_{\infty}, \omega, p(\omega)\right)\right|=\left|c_{\infty}(\lambda) \mu_{\infty}^{+}(\lambda)\left(1-\mu_{\infty}^{+}(\lambda)\right)^{-1}\right| \cdot\left|W_{\infty}(\lambda, \omega)\right|^{2} .
$$

Функция

$$
\lambda \rightarrow W_{\infty}(\lambda, \omega)
$$

аналитична по $\lambda$ как функция со значениями в $C(\Omega)$ и в силу $(27)$ в точке $\lambda_{\infty}$ имеет нуль. Функция $\lambda \rightarrow\left(1-\mu_{\infty}^{+}(\lambda)\right)$ в точке $\lambda_{\infty}$ имеет нуль первого порядка, что и доказывает лемму.

Из доказанной леммы следует, что

$$
\lim _{\left|\lambda-\lambda_{\infty}\right| \rightarrow+0} \int\left|t_{\infty}^{+}(\lambda, \omega, p(\omega))_{\mathrm{reg}}-t_{\infty}^{+}(\lambda, \omega, p(\omega))\right| d \omega=0 .
$$

Приступим к изложению основного результата.

Положим по определению

$$
\text { v.p. } t_{\infty}^{+}\left(\lambda_{\infty}, \omega, p(\omega)\right)=\lim _{\left|\lambda-\lambda_{\infty}\right| \rightarrow+0} t_{\infty}^{+}(\lambda, \omega, p(\omega)) .
$$

Сушествование этого предела вытекает из предыдущей леммы.

ТЕОРема 1. Пусть $\nu_{n}-$ последовательность действительных чисел, которая удовлетворяет условию

$$
\left|\nu_{n}-\operatorname{Re} \lambda_{n}\right|=O\left(\left(\operatorname{Im} \lambda_{n}\right)^{1+\varepsilon}\right) \quad \varepsilon>0, \quad n \rightarrow \infty .
$$

Тогда справедливы равенства

$$
\begin{array}{r}
\lim _{n \rightarrow \infty} \int \mid t_{n}^{+}\left(\nu_{n}, \omega, p(\omega)\right)-\text { v.p. } t_{\infty}^{+}\left(\lambda_{\infty}, \omega, p(\omega)\right) \mid d \omega=\frac{|\beta|}{\pi}, \\
\lim _{n \rightarrow \infty} \int\left|t_{n}^{+}\left(\nu_{n}, \omega, p(\omega)\right)-t_{\infty}^{+}\left(\nu_{n}, \omega, p(\omega)\right)\right| d \omega=\frac{|\beta|}{\pi},
\end{array}
$$

әде $\beta$ - константа из (12).

ДокАЗАТЕЛЬСтво. Заметим, что утверждения (37) и (38) эквивалентны в силу определения (36).

Справедливы равенства

$$
\begin{aligned}
t_{n}^{+}\left(\nu_{n}, \omega, p(\omega)\right)-t_{n}^{+}\left(\nu_{n}, \omega, p(\omega)\right)_{\infty}=[ & \left.t_{n}^{+}\left(\nu_{n}, \omega, p(\omega)\right)-t_{n}^{+}\left(\nu_{n}, \omega, p(\omega)\right)_{\mathrm{reg}}\right]_{1} \\
& +\left[t_{n}^{+}\left(\nu_{n}, \omega, p(\omega)\right)_{\mathrm{reg}}-t_{\infty}^{+}\left(\nu_{n}, \omega, p(\omega)\right)_{\mathrm{reg}}\right]_{2} \\
& +\left[t_{\infty}^{+}\left(\nu_{n}, \omega, p(\omega)\right)_{\mathrm{reg}}-t_{n}^{+}\left(\nu_{n}, \omega, p(\omega)\right)_{\infty}\right]_{3} .
\end{aligned}
$$


Из леммы 6 следует, что

$$
\lim _{n \rightarrow \infty} \sup _{\omega}\left|[\ldots]_{2}\right|=0
$$

а из леммы 7 вытекает, что

$$
\lim _{n \rightarrow \infty} \sup _{\omega}\left|[\ldots]_{3}\right|=0 .
$$

Из леммы $4,(11)$ и (33) следует, что

$$
\begin{aligned}
\int\left|[\ldots]_{1}\right| d \omega & =\left|c_{n}\left(\nu_{n}\right)\left(1-\mu_{n}\left(\nu_{n}\right)\right)^{-1}\right| \int\left|W_{n}\left(\nu_{n}, \omega\right)\right|^{2} d \omega \\
& =\frac{1}{\pi}\left|c_{n}\left(\nu_{n}\right)\left(1-\mu_{n}\left(\nu_{n}\right)\right)^{-1}\right|\left|\left\langle\psi_{n}\left(\nu_{n}\right), V_{n} \psi_{n}\left(\nu_{n}\right)\right\rangle \operatorname{Im} \mu_{n}^{+}\left(\nu_{n}\right)\right| .
\end{aligned}
$$

Справедливы соотношения

$$
\begin{gathered}
1-\mu_{n}^{+}\left(\nu_{n}\right)=\mu_{n}^{+}\left(\lambda_{n}\right)-\mu^{+}\left(\nu_{n}\right)=i \frac{d \mu_{n}^{+}\left(\nu_{n}\right)}{d \lambda} \operatorname{Im} \lambda_{n}+O\left(\left(\operatorname{Im} \lambda_{n}\right)^{1+\varepsilon}\right), \\
\operatorname{Im} \mu_{n}^{+}\left(\nu_{n}\right)=\operatorname{Im}\left(i \frac{d \mu_{n}^{+}\left(\nu_{n}\right)}{d \lambda}\right) \operatorname{Im} \lambda_{n}+O\left(\left(\operatorname{Im} \lambda_{n}\right)^{1+\varepsilon}\right) .
\end{gathered}
$$

Из формулы (24) следует, что производная

$$
\left.\frac{d \mu_{\infty}^{+}(\lambda)}{d \lambda}\right|_{\lambda=\lambda_{\infty}}
$$

- действительное число, поэтому из равенств (43) и (44) вытекает, что

$$
\lim _{n \rightarrow \infty}\left|\operatorname{Im} \mu_{n}^{+}\left(\nu_{n}\right)\left(1-\mu_{n}^{+}\left(\nu_{n}\right)\right)^{-1}\right|=1 .
$$

Далее мы замечаем, что

$$
\lim _{n \rightarrow \infty}\left|c_{n}\left(\nu_{n}\right)\left\langle\psi_{n}\left(\nu_{n}\right), V_{n} \psi_{n}\left(\nu_{n}\right)\right\rangle\right|=\left|\frac{\left\langle\psi_{\infty}, V_{\infty} \psi_{\infty}\right\rangle}{\left(\left\langle l^{-1}\left(\psi_{\infty}\right), V_{\infty} \psi_{\infty}\right\rangle\right)}\right|=|\beta| .
$$

Из (39)-(46) следует утверждение теоремы.

ТЕОРемА 2. Числа $\lambda_{n}$ являются полюсами функиии

$$
\lambda \rightarrow t_{n}^{+}(\lambda, \omega, p(\omega)),
$$

рассматриваемой как функиия со значениями в $C(\Omega)$, и поэтому являются полюсами матрицы рассеяния.

ДокАЗАТЕльство. Если бы существовала такая последовательность $\lambda_{n_{j}}$, что в $(33)$ было бы выполнено тождество

$$
\forall j \quad\left\langle V_{n_{j}} l^{-1}\left(\psi_{n_{j}}\left(\lambda_{n_{j}}\right)\right), U^{-1}\left(\lambda_{n_{j}}, p(\omega)\right)\right\rangle U\left(V_{n_{j}} \psi_{n_{j}}\right)\left(\lambda_{n_{j}}, \omega\right) \equiv 0,
$$

то последовательность

$$
j \rightarrow t_{n_{j}}^{+}(\lambda, \omega, p(\omega))
$$

была бы последовательностью аналитических в круге $\left|\lambda-\lambda_{\infty}\right|<\delta$ функций со значениями в $C(\Omega)$, которая сходится равномерно на границе круга и, следовательно, сходится равномерно внутри круга, что противоречит теореме 1. 
5. Заключительные замечания. Отличие нашей модели от модели, предложенной Лифшицем, состоит в том, что Лифшиц рассматривал матрицу рассеяния $S\left(A_{n}, A_{\infty}\right)$, а мы рассматриваем матрицу рассеяния $S\left(A_{n}, A_{0}\right)$.

Из численных экспериментов хорошо известно, что при рассеянии на квазиуровне четко выраженного симметричного квантового колодца (quantum well) независимо от деталей потенциала коэффициент прохождения достигает единицы, а если колодец не симметричен, то этого нет. Математическая модель этого явления предложена в [6]. Для рассмотренной в [6] модели отображение $U$ есть преобразование Фурье, пространство $\Omega$ есть пара точек $-1,+1$. Отображения $l$ и $p$ в случае потенциала общего вида даются формулами

$$
l(\psi)(x)=\bar{\psi}(x), \quad p(-1)=1, \quad p(1)=-1 .
$$

Если же потенциал симметричен, то появляется дополнительная возможность: можно положить

$$
l(\psi)(x)=\bar{\psi}(-x), \quad p(-1)=-1, \quad p(1)=1 .
$$

Подробности см. в [6]. Оказывается, что аналогичное явление происходит и в других рассмотренных нами случаях (при рассеянии на резонаторе Гельмгольца, на ловушечном потенциале, на скачках коэффициента преломления для волнового уравнения), где речь идет о резонансе на возмущенном собственном значении из непрерывного спектра.

Эта работа была выполнена во время стажировки автора в Чалмерсе (Department of Mathemetics, Chalmers University of Technology and Göteborg University). Я благодарен руководству университетов за предоставленные условия для работы и благодарен проф. Лейфу Аркериду и проф. Алексею Гейнцу (Leif Arkeryd and Alexei Heintz) за гостеприимство. Первоначальньй вариант работы опубликован в [7].

\section{СПИСОК ЦИТИРОВАННОЙ ЛИТЕРАТУРЫ}

[1] Базь А. И., Зельдович Я. Б., Переломов А. М. Рассеяние, реакции и распады в нерелятивисткой квантовой механике. М.: Наука, 1971.

[2] Бирман М.Ш., Энтина С. Б. Стационарньй подход в абстрактной теории рассеяния // Изв. АН СССР. Сер. матем. 1967. Т. 31. С. 401-430.

[3] Яфаев Д.Р. Математическая теория рассеяния. С.-Пб.: Изд-во С.-Петербургского ун-та, 1994.

[4] Howland J.S. The Livsic matrix in perturbation theory // J. of Math. Anal. Appl. 1984. V. 101. P. 491-513.

[5] Albeverio S., Hoegh-Kron R. Perturbations of resonances in quantum mechanics // J. Math. Anal. Appl. 1984. V. 101. P. 491-513.

[6] Арсеньев А.А. Резонансные свойства матрицы рассеяния для одномерного оператора Шредингера с ловушечным потенциалом // Матем. сб. 1996. Т. 187. №6. С. 3-20.

[7] Arsen'ev A. A. Model of Resonance Scattering. Preprint № 2000:15. Department of Math., Chalmers Univ. of Technology and Göteborg University, 2000. 